\title{
Diabetes e Doenças Auto-Imunes: Prevalência de Doença Celíaca em Crianças e Adolescentes Portadores de Diabetes Melito Tipo 1
}

\begin{abstract}
RESUMO
Objetivo: Determinar a prevalência de doença celíaca em crianças e adolescentes portadores de diabetes melito tipo1 (DM1) no Instituto Estadual de Diabetes e Endocrinologia Luiz Capriglione (IEDE). Métodos: Foram analisadas amostras de sangue de 120 crianças e adolescentes portadores de DM1 do Ambulatório de Diabetes do IEDE para a pesquisa do anticorpo antitransglutaminase tecidual humana IgA e dosagem da IgA sérica. Aqueles com sorologia positiva foram encaminhados para endoscopia digestiva alta com biópsia de intestino delgado para a confirmação da doença celíaca. Resultados: $O$ anticorpo foi positivo em três dos 120 pacientes analisados. Todos os positivos apresentaram biópsia de intestino delgado confirmatória para doença celíaca, gerando prevalência desta doença no grupo estudado de 2,5\%. Conclusão: A prevalência de doença celíaca encontra-se aumentada entre crianças e adolescentes com DM1 acompanhadas no IEDE quando comparadas à normalidade. Como a maioria é assintomática, recomenda-se o rastreamento periódico desta doença em todas as crianças com DM1. (Arq Bras Endocrinol Metab 2008; 52/9:1461-1465)
\end{abstract}

Descritores: Prevalência; Doenças auto-imunes; Diabetes melito tipo 1; Doença celíaca

\begin{abstract}
Diabetes and Autoimmune Diseases: Prevalence of Celiac Disease in Children and Adolescents with Type 1 Diabetes.

Objective: Determinate the prevalence of celiac disease in children and adolescents with type 1 diabetes mellitus (DM1) in attendance in Instituto Estadual de Diabetes e Endocrinologia Luiz Capriglione (IEDE). Methods: Blood samples were analyzed in 120 children and adolescents with DM1 from IEDE Diabetes Clinic for the $\lg \mathrm{A}$ antitissue-transglutaminase antibody and dosage of the seric $\lg A$. Those with positive serology were guided for upper endoscopy with small-bowel biopsy to confirm the celiac disease. Results: The antibody was positive in 3 of the 120 patients. The small-bowel biopsy was confirmatory in all of the positive patients, leading to a prevalence of celiac disease of $2.5 \%$ in the studied group. Conclusions: The prevalence of celiac disease is increased in children and adolescents with DM1 when compared with normality. As most are asymptomatic, it is recommended periodical screening of celiac disease in children with DM1. (Arq Bras Endocrinol Metab 2008; 52/9:1461-1465)
\end{abstract}

Keyword: Prevalence; Autoimmune disorders; Type 1 diabetes mellitus; Celiac disease

\section{artigo original}

\author{
Camila Mont-Serrat \\ Claudio HoinefF \\ RICARDO M. R. MEIRELLES \\ ROSANE KUPFER
}

Departamento de Diabetes do Instituto Estadual de Diabetes e Endocrinologia Luiz Capriglione, Rio de Janeiro, RJ, Brasil. 


\section{INTRODUÇÃO}

associação entre diabetes melito tipo l (DMl) e outras doenças auto-imunes, particularmente as desordens tireoidianas auto-imunes e a doença celíaca, é conhecida de longa data. A prevalência de doença celíaca entre portadores de DMl tem sido estimada em cerca de $4 \%$, com variação entre $0 \%$ e $10,4 \%$ (1), superando, em muito, a prevalência da população geral $(0,5 \%$ e $1 \%)$. Suscetibilidade genética parece ser o principal fator para esta associação. Atualmente sabese que o principal determinante desta suscetibilidade é a região genômica denominada HLA, caracterizada pela seqüência de genes localizada no cromossomo 6p21. HLA DR3-DQ2 e DR4-DQ8 são compartilhados pelo DMl, doença celíaca e outras doenças auto-imunes.

A maioria das crianças com DMl e doença celíaca apresenta formas silenciosas ou subclínicas da doença. Sintomas clássicos, como diarréia e dor abdominal, podem não estar presente em muitas dessas crianças. Deficiência de ferro e ácido fólico, com ou sem anemia (1), baixa estatura (1), baixo peso (2), retardo puberal (2), atraso na maturação óssea (2), diabetes de difícil controle e episódios freqüentes de hipoglicemia $(2,3)$ podem ser as únicas manifestações da doença. Infelizmente, poucos médicos pensam na doença celíaca como causa destes sinais e sintomas e muitos acabam por atribuí-los ao mau controle glicêmico ou, até mesmo, a doenças tireoidianas ou adrenais coexistentes. Dessa forma, o diagnóstico é retardado em anos, promovendo consequiências deletérias para estas crianças.

Com base nestes dados, estudos demonstram a importância da realização do rastreamento periódico da doença celíaca em todas as crianças com DMl. A triagem inicial tem sido realizada por meio de testes sorológicos, sendo o anticorpo antiendomísio $\operatorname{IgA} \mathrm{e}$ antitransglutaminase tecidual humana IgA os mais utilizados. Aqueles com sorologia positiva devem ter o diagnóstico confirmado por biópsia de intestino delgado por meio de endoscopia digestiva alta (1-8). Deve ser considerada também a dosagem da IgA sérica durante o rastreamento, visto que pequeno percentual de pacientes com doença celíaca é portador de deficiência seletiva de IgA, apresentando negatividade para estes anticorpos.

O objetivo deste estudo é estimar a prevalência de doença celíaca em crianças e adolescentes portadores de DMl em acompanhamento no Ambulatório de
Diabetes do Instituto Estadual de Diabetes e Endocrinologia Luiz Capriglione (IEDE).

\section{MATERIAIS E MÉTODOS}

O estudo foi realizado no IEDE, no Rio de Janeiro, no período de março a julho de 2007. O protocolo foi aprovado pelo Comitê de Ética em Pesquisa do IEDE, e todos os responsáveis dos pacientes assinaram o termo de consentimento livre e esclarecido.

Foi obtida amostra de 120 pacientes portadores de DMl, de maneira consecutiva, na ordem de apresentação espontânea, nas consultas de rotina do Ambulatório de Diabetes previamente agendadas. Foram selecionados pacientes de ambos os sexos e com idade entre 0 e 18 anos, independentemente da presença de sintomas gastrintestinais. Foram coletadas amostras de sangue para a pesquisa do anticorpo antitransglutaminase tecidual humana IgA e dosagem da IgA sérica. Os testes foram realizados no Laboratório de Diagnósticos da América, por meio dos métodos Elisa e nefelometria, respectivamente. Foram consideradas sorologias positivas aquelas com títulos de anticorpos antitransglutaminase tecidual humana maior que $35 \mathrm{UA} / \mathrm{mL}$. A deficiência de IgA seria confirmada quando os níveis de IgA sérica encontravam-se menores que $5 \mathrm{mg} / \mathrm{dL}$. Os pacientes com sorologia positiva para o antitransglutaminase foram convocados para a realização de endoscopia digestiva alta com biópsia de intestino delgado, realizada no Hospital Universitário Clementino Fraga Filho (HUCFF), nos maiores de 14 anos, e no Instituto de Pediatria e Puericultura Martagão Gesteira (IPPMG), nos menores de 14 anos. Foram considerados portadores de doença celíaca apenas aqueles com sorologia positiva e biópsia de intestino delgado compatível com doença celíaca. Os dados clínicos, como controle glicêmico, peso, altura, índice de massa corporal (IMC) e sintomas gastrintestinais foram avaliados em todos os pacientes. Aqueles com diagnóstico confirmado de doença celíaca tiveram seus prontuários analisados para informações complementares.

Os dados estatísticos descritivos utilizados foram a mediana e a amplitude, pois os valores analisados não se distribuíam conforme a curva normal.

\section{RESULTADOS}

A Tabela 1 resume as características clínicas e laboratoriais da população estudada. 
Tabela 1. Características da população estudada.

\begin{tabular}{ll}
$\mathbf{n}$ & 120 \\
\hline Meninos & $57,5 \%$ \\
\hline Idade* (anos) $^{-11,58(3-18,42)}$ \\
\hline Idade ao diagnóstico do DM* (anos) & $8,33(0,75-16,42)$ \\
\hline Tempo de DM* (anos) & $2,25(0,25-16,75)$ \\
\hline HbAlc* (VR = 4\% a 6\%) & $8,7 \%(4,9-19,7 \%)$ \\
\hline Sintomas gastrintestinais & $38,3 \%$ \\
\hline Hipoglicemia auto-referida & $77,5 \%$ \\
\hline Deficiência de lgA & 0 \\
\hline Antitransglutaminase positivo & $3(2,5 \%)$ \\
\hline
\end{tabular}

* Mediana e amplitude.

$\mathrm{O}$ anticorpo antitransglutaminase tecidual humano foi positivo em três dos 120 pacientes analisados. Todos os positivos foram submetidos à endoscopia digestiva alta com biópsia para confirmação da doença. A biópsia de intestino delgado foi confirmatória para doença celíaca nos três pacientes com sorologia positiva, gerando prevalência de $2,5 \%$ para esta doença no grupo estudado. Todos eram do sexo masculino. A Tabela 2 descreve as características dos pacientes com diagnóstico confirmado de doença celíaca. O paciente 1 era um menino da raça negra, de 3 anos de idade, com episódios freqüentes de diarréia, dor e distensão abdominal, e portador de anemia microcítica e hipocrômica com valores de VCM e HCM baixos. Foi afastada a hemoglobinopatia como etiologia da anemia por meio de eletroforese de hemoglobina. $\mathrm{Na}$ análise da cinética do ferro, o ferro sérico e a ferritina eram baixos, sugerindo deficiência de ferro como provável etiologia da anemia. $\mathrm{Na}$ análise de seu gráfico de crescimento, apresentavase abaixo do alvo genético familiar e com curva peso versus idade retificada. Sua idade óssea era compatível com a idade cronológica ( 3 anos e 6 meses).

O paciente 2 tinha 17 anos e referia apenas episódios esporádicos de dor abdominal ao ingerir alimentos gordurosos. Sua altura encontrava-se dentro do alvo familiar esperado, e não tinha anemia. Sua idade óssea era compatível com a idade cronológica (18 anos). Seu controle glicêmico também era inadequado, com hemoglobina glicada elevada $(9,9 \%)$ e hipoglicemias freqüentes. $\mathrm{O}$ controle glicêmico era difícil, com hemoglobina

Tabela 2. Pacientes positivos para doença celíaca.

\begin{tabular}{|c|c|c|c|}
\hline & 1 & 2 & 3 \\
\hline Idade/Sexo & 3 anos/masculino & 17 anos/masculino & 11 anos/masculino \\
\hline Idade ao diagnóstico do diabetes melito & 1 ano e 7 meses & 12 anos & 10 anos \\
\hline Duração do diabetes melito & 1 ano e 5 meses & 5 anos & 1 ano \\
\hline Peso ao nascimento & $2,750 \mathrm{~kg}$ & $3,840 \mathrm{~kg}$ & $1,230 \mathrm{~kg}^{*}$ \\
\hline Diarréia & Sim & Não & Não \\
\hline Dor abdominal & Sim & Sim & Não \\
\hline Distensão abdominal & Sim & Não & Não \\
\hline Déficit de crescimento & Sim & Não & Não \\
\hline Hipoglicemia & $4-5$ vezes/semana & 2-3 vezes/semana & 3 vezes/semana \\
\hline $\mathrm{HbAlc}$ & $9,4 \%$ & $9,9 \%$ & $8,3 \%$ \\
\hline $\mathrm{Hb}$ & $11 \mathrm{~g} / \mathrm{dL}$ & $15,2 \mathrm{~g} / \mathrm{dL}$ & $14 \mathrm{~g} / \mathrm{dL}$ \\
\hline $\mathrm{Ht}$ & $36 \%$ & $44 \%$ & $41,8 \%$ \\
\hline VCM & $66 \mathrm{fl}$ & $85 \mathrm{fl}$ & $84,4 \mathrm{fl}$ \\
\hline TSH & $5,59 \mathrm{mclU} / \mathrm{ml}$ & $2,42 \mathrm{mclU} / \mathrm{ml}$ & $2,41 \mathrm{mclU} / \mathrm{ml}$ \\
\hline T4L & $1,08 \mathrm{ng} / \mathrm{dL}$ & $1,61 \mathrm{ng} / \mathrm{dL}$ & $1,37 \mathrm{ng} / \mathrm{dL}$ \\
\hline Anti-TPO & Negativo & Negativo & Positivo \\
\hline Antitransglutaminase & $75 \mathrm{UA} / \mathrm{ml}$ & $67 \mathrm{UA} / \mathrm{ml}$ & $73,5 \mathrm{UA} / \mathrm{ml}$ \\
\hline Deficiência de lgA & Não & Não & Não \\
\hline Observação & & & Tratamento de hipotireoidismo \\
\hline
\end{tabular}


glicada de $9,4 \%(V R=4 \%$ a $6 \%)$ e episódios freqüentes de hipoglicemia.

O paciente 3 era uma criança de 11 anos, portadora de hipotireoidismo por tireoidite de Hashimoto, que não referia nenhum sintoma gastrintestinal sugestivo de doença celíaca. Também se encontrava com bom desenvolvimento pôndero-estatural, mantendo-se dentro do alvo genético esperado e com idade óssea de 13 anos. Tinha hemoglobina glicada fora do alvo terapêutico $(8,3 \%)$, episódios freqüentes de hipoglicemia e não era portador de anemia.

Cerca de $38,3 \%$ dos pacientes entrevistados apresentavam sintomas gastrintestinais comuns à doença celíaca, como diarréia, dor e distensão abdominal, porém destes apenas dois $(4,3 \%)$ apresentaram positividade para a doença.

\section{DISCUSSÃo}

A doença celíaca é uma desordem crônica intestinal causada pela hipersensibilidade ao glúten presente no trigo, centeio e cevada, em pessoas geneticamente predispostas. Há o desenvolvimento de enteropatia inflamatória com hiperplasia de criptas e atrofia das vilosidades intestinais, levando à má-absorção dos alimentos. Sua prevalência na população geral oscila entre $0,5 \%$ e $1 \%$, afetando um a cada 120 pessoas na Europa e na América do Norte (3). Entre os diabéticos tipo 1, esta prevalência pode variar de $0 \%$ a $10 \%$, com relatos até de $21 \%$ (4), representando risco 20 vezes maior de doença celíaca neste grupo de pacientes (9). No presente estudo, encontrou-se prevalência de $2,5 \%$, confirmando a maior associação entre essas duas doenças.

As crianças portadoras de doença celíaca podem apresentar diarréia, atraso de crescimento, fraqueza muscular, edema, anemia e raquitismo. No entanto, a maioria dos pacientes com DMl e doença celíaca refere pouco ou nenhum sintoma relacionado à má-absorção, e quando presentes, geralmente são leves e observados apenas retrospectivamente (9). No estudo realizado por Barera e cols. (9) na Itália, apenas dois dos 16 pacientes positivos para doença celíaca durante o rastreamento tinham queixas sugestivas da doença. No presente estudo, apenas um dos três pacientes com diagnóstico confirmado de doença celíaca apresentava sintomas graves. A absorção dos nutrientes pode ser variável, alterando as necessidades de insulina e afetando o controle do diabetes (5). Episódios inexplicados de hipoglicemia devem alertar para a possibilidade da coexistência desta doença nos pacientes com DMl (3). O desenvolvimento pôndero-estatural também pode ser afetado, sendo causa de baixa estatura e baixo peso nestes pacientes. Muitas vezes o impacto no crescimento pode se tornar aparente apenas após o início da dieta sem glúten, com melhora no percentil de crescimento (5). Já foi demonstrado aumento do IMC após o início da dieta isenta de glúten, o que pode ocorrer em parte pela própria melhora no controle do diabetes com o tratamento (5). Anemia, por deficiência de ferro e/ou ácido fólico e deficiência de vitaminas são as anormalidades laboratoriais mais comuns entre estes pacientes (5). No presente estudo, um dos pacientes (paciente 1) com diagnóstico confirmado de doença celíaca apresentava anemia por deficiência de ferro.

A doença celíaca, sintomática ou não, está associada a outras doenças e condições graves, que são atenuadas ou eliminadas com a instituição da dieta isenta de glúten. Linfoma de Hodgkin e adenocarcinoma intestinal são mais comuns entre os celíacos do que na população geral (6), e o risco destas complicações parece ser proporcional ao tempo de exposição ao glúten (10). Como há fortes evidências de que a dieta isenta de glúten reverte ou pelo menos diminui o risco destas complicações, é recomendado o diagnóstico precoce com a pronta instituição da dieta sem glúten em todos os pacientes diabéticos tipo 1 com doença celíaca, mesmo que assintomática, a fim de prevenir o desenvolvimento destas e outras complicações (9). O estudo realizado por Hansen e cols. (11), na Dinamarca, teve como objetivo demonstrar os benefícios da dieta isenta de glúten nestes pacientes. Neste estudo, 31 pacientes com ambas as doenças foram observados pelo período de, pelo menos, dois anos enquanto consumiam dieta isenta de glúten. Durante o seguimento, os pacientes sintomáticos apresentaram melhora do bem-estar, a maioria ganhou peso e os menores de 14 anos apresentaram aumento significativo da estatura. Houve aumento importante da hemoglobina, VCM e ferritina sérica após os dois anos de dieta. Porém, neste estudo, assim como em outros (10), a HbAlc praticamente não sofreu alteração nos pacientes em dieta sem glúten. No entanto, alguns pacientes apresentaram diminuição dos episódios de hipoglicemia após o início da dieta, propiciando melhora na qualidade de vida desses pacientes.

A alta prevalência da doença celíaca em crianças com DMl, a importância da prevenção de suas complicações e o potencial destas complicações serem contro- 
ladas com a instituição de dieta isenta de glúten suportam a recomendação da realização de rastreamento para a doença celíaca em todos os casos de DMl (12). O rastreamento pode revelar casos subclínicos da doença. Porém, a freqüência e a forma da realização do rastreamento ainda não estão plenamente estabelecidos. Os primeiros anticorpos utilizados foram antigliadina $\operatorname{IgA}$ e $\operatorname{IgG}$. Por apresentarem sensibilidade e especificidade inferiores, o antigliadina IgA é um marcador sorológico útil apenas em crianças sintomáticas menores de 2 anos, e o antigliadina IgG nos $2 \%$ a $20 \%$ dos pacientes celíacos com deficiência de $\operatorname{IgA}(3)$. O anticorpo antitransglutaminase tecidual humana IgA tem sido recomendado como o de escolha (10) por apresentar sensibilidade superior ao antiendomísio IgA, não obstante a especificidade semelhante. Para evitar o encontro de falso-negativos para estes anticorpos, é recomendada a verificação dos níveis séricos de $\operatorname{IgA}$ durante o rastreamento $(10,13)$. Aqueles pacientes que apresentam sorologia positiva devem ser submetidos à endoscopia digestiva alta com biópsia de intestino delgado para a confirmação da doença. Aqueles que apresentarem sorologia negativa devem ser observados e ter novo rastreamento realizado periodicamente $(7,12)$. $\mathrm{O}$ intervalo apropriado entre as reavaliações ainda não está definido. Um estudo realizado na Itália (12) demonstrou que $75 \%$ das crianças e adolescentes tiveram o diagnóstico de doença celíaca próximo de quatro anos de duração do diabetes. Observaram tendência à diminuição da prevalência conforme aumentava a duração do diabetes. Segundo este estudo, doença celíaca é raramente encontrada após dez anos do diagnóstico do diabetes. Com base nestes achados, o estudo recomenda o rastreamento anual das crianças assintomáticas durante os primeiros quatro anos de diabetes e aconselha continuá-lo até pelo menos dez anos, com intervalos dependendo das condições econômicas do local.

Conclui-se que a alta prevalência de doença celíaca em crianças com DMl e a ocorrência de complicações preveníveis e tratáveis apóiam a recomendação do rastreamento periódico desta doença independentemente da presença ou não de sinais e/ou sintomas sugestivos de doença celíaca.

Agradecimentos: Agradecimento especial ao Laboratório Diagnósticos da América, que tornou possível a idealização deste estudo por meio da realização dos testes sorológicos; e ao Dr. Antonio Calçado e Dr. César Augusto Amorim, que realizaram as endosco- pias digestivas altas. Os autores declaram não haver conflitos de interesse científico neste artigo.

\section{REFERÊNCIAS}

1. Collin P, Kaukinen K, Valimaki M, Salmi J. Endocrinological disorders and celiac disease. Endocr Rev. 2002;23(4):464-83.

2. Screening for celiac disease in children with type 1 diabetes. Diabetes Care. 2003;26(6):1932-9.

3. Farrell RJ, Kelly CP. Celiac sprue. N Engl J Med. 2002;346 (3):180-8.

4. Brandt KG, Silva GAP, Antunes MMC. Doença celíaca em um grupo de crianças e adolescentes portadores de diabetes mellitus tipo 1. Arq Bras Endocrinol Metab. 2004;48(6):823-7.

5. Book LS. Diagnosing celiac disease in 2002: who, why, and how? Pediatrics. 2002;109:952-4.

6. Nelsen DAJ. Gluten-sensitive enteropathy (celiac disease): more common than you think. Am Fam Physician. 2002;66: 2259-66.

7. Glastras SJ, Craig ME, Verge CF, Chan AK, Cusumano JM, Donaghue $\mathrm{KC}$. The role of autoimmunity at diagnosis of type 1 diabetes in the development of thyroid and celiac disease and microvascular complications. Diabetes Care. 2005;28:2170-5.

8. Barker JM. Clinical review: type 1 diabetes-associated autoimmunity: natural history, genetic associations, and screening. $J$ Clin Endocrinol Metabol. 2006;91(4):1210-7.

9. Barera G, Bonfanti R, Viscardi M, Bazzigaluppi E, Calori G, Meschi F, et al. Occurrence of celiac disease after onset of type 1 diabetes: a 6 -year prospective longitudinal study. Pediatrics. 2002;109:833-8.

10. Peretti N, Bienvenu F, Bouvet $C$, Fabien N, Tixier F, Thivolet $C$, et al. The temporal relationship between the onset of type 1 diabetes and celiac disease: a study based on immunoglobulin a antitransglutaminase screening. Pediatrics. 2004;113; 418-22.

11. Hansen D, Brock-Jacobsen B, Lund E, Bjorn C, Hansen L, Nielsen $C$, et al. Clinical benefit of a gluten-free diet in type 1 diabetic children with screening-detected celiac disease. Diabetes Care. 2006;29:2452-6.

12. Cerutti F, Bruno G, Chiarelli F, Lorini R, Meschi F, Sacchetti C, et al. Younger age at onset and sex predict celiac disease in children and adolescents with type 1 diabetes. Diabetes Care. 2004;27:1294-8.

13. Doolan A, Donaghue K, Fairchild J, Wong M, Williams AJ. Use of HLA typing in diagnosing celiac disease in patients with type 1 diabetes. Diabetes Care. 2005;28:806-9.

Endereço para correspondência:

Camila Mont-Serrat

Praça Restier Gonçalves, 115, apto. 303, Recreio dos Bandeirantes

22790-180 Rio de Janeiro RJ

E-mail: camserrat@yahoo.com.br 